Fiona Smith

Professional lead for children and young people's nursing,

Royal College of Nursing, United Kingdom and co-ordinator of the Paediatric

Nursing Associations of Europe

\title{
Priorities for children and young people - opportunities and challenges for children and young people's nurses
}

Across Europe children's nurses today face many challenges, including rising childhood obesity, the soaring incidence of issues with the mental health of children and young people, the effects of social media, child maltreatment and the impact of poverty, war and conflict on children and families. There are opportunities for children's nurses to undertake new roles and to influence both policy and practice to improve the health outcomes of children and young people, and thereby the future health of the population.

The role of children's nurses continues to expand in response to the changing healthcare needs of children and young people. Nursing roles have developed to maximise the use of nursing knowledge and skills in the delivery of healthcare services. Children's nurses provide care across many settings and not just in traditional children's wards, but in social care and education settings, as well as in children's own homes. Today children's nurses in many countries work in a variety of ways to enable children with complex healthcare needs, for example, to be cared for at home, support parents in the early years of a child's life and provide palliative care, symptom management and end of life care to enable a child to live as near a normal life as possible and to die at home. Children's nurses working in advanced roles assess, diagnose, treat, prescribe and discharge patients independently. Specialist children's nurses may undertake surgical pre-assessment and provide complete follow-up care to children with long-term conditions such as asthma and eczema. Other senior and experienced practitioners spend some of their working week as part of medical rotas in highly technical and specialist areas, such as neonatal and children's intensive care, while others in nurse consultant roles may lead entire teams in the provision of a service for children on long-term ventilation.

The presentation will highlight key challenges for children's nurses today, drawing upon examples to demonstrate the changing role of children's nurses, as well as to highlight differences across Europe. The work of the Paediatric Nursing Associations of Europe will also be highlighted and the need to learn from each other to achieve improvements in children and young people's health outcomes. 